\title{
Dynamic Nonlinear Correlation Studies on Stock and Oil Market Based on Copula
}

\author{
He Xin ${ }^{1, *}$ and Zhang Guofu ${ }^{2}$ \\ ${ }^{1}$ Department of Information Management, Beijing Institute of Petrochemical Technology, Beijing, 102617, China; \\ ${ }^{2}$ Department of Information Management, Tianjin University of Science and Technology, Tianjin, 300457, China
}

\begin{abstract}
Employing the dataset of WTI oil spot price and stock price index in China, Brazil, India, US, German, France, UK and Japan, this paper obtains five subintervals of whole sample range through a nonparametric multiple change point algorithms. Furthermore, it analyzes dependence between oil spot price and stock price index through copula model and computes the value of VaR and ES based on simulation for every subinterval. It reveals that dependence between oil spot price and stock price index during financial crisis is an asymmetric tail dependence. The value of VaR and ES of the oil spot price and stock price index shows irregular fluctuation.
\end{abstract}

Keywords: Copula, Dependence, Oil, Stock, Nonparametric, Price.

\section{INTRODUCTION}

Over the years, researches have been conducted on the connection between price changes in the oil and the stock markets. The results, however, vary substantially due to different models and objects used.

Apergis and Miller analyzed the impact of oil price shocks on the stock markets of 8 developed countries [1], and found there is no significant impact in this respect. Park and Ratti studied the connections between oil price shocks and stock markets of the United States and 13 European countries [2], and concluded that oil price shocks have significant impact on these stock markets. Nandha and Faff examined data from 35 global industries [3], and confirmed that oil price shocks have significant negative impact on all of the industries except oil and natural gas. Killian and Park discovered that the impact of oil price shocks on the stock market may vary depending on the causes of such shocks [4]. Mohanty et al., studied the impact of oil price changes on the stock prices of oil and gas companies in the central and eastern Europe [5], reporting that no significant connection was found during the research period. Driesprong analyzed the connection between oil price changes and returns of key stock markets around the world [6], and found that worldwide oil price shocks may be an indicator of returns of stock markets, particularly those of developed countries.

In China, there have been many researchers on the connections between oil price shocks and the stock market. Using Bivariate GARCH and VAR models, Jin Hongfei and Jin Luo studied oil prices and returns of the stock markets in China and United States [7], as well as their spillover effect. They found that oil price shocks have negative spillover effect on the return of US stock market and there is a two-way

*Address correspondence to this author at the Department of information management, Beijing Institute of Petrochemical Technology, Beijing, 102617, China; Tel: +86 10 52489515; Fax: +86 10 58850501-1987; E-mail: hexin@bipt.edu.cn volatility spillover effect. With regard to the Chinese stock market, there is no spillover effect on either the return or the volatilities of any direction. Using Granger Causality Analysis, the VAR model, Impulse Response Function (IRF), Forecast Error Variance Decomposition (FEVD) and the MGARCH-BEKK $(1,1)$ model, Liu Xiangyun and Zhu Chunming analyzed the volatility spillover and mean spillover effects of log returns of NYSE as of WTI spot date and Shanghai Stock Exchange as of its index date [8]. They found that the risk spillover between returns of both markets had been marginal and volatile until 2007, when the spillover effect became significant. Jin Hongfei and Jin Luo used the Bivariate GED-GARCH $(1,1)$-M model to analyze the impact of international oil price changes on returns of stocks of 14 different sectors in China [9], finding positive impact on the oil and natural gas sectors, notably negative impact on the utilities, travel and leisure, personal and household appliance, healthcare, finance, building materials automotive and parts sectors, and no significant impact on other sectors. Using VAR and GARCH-BEKK models, Zhou Detian and Guo Jinggang analyzed the connections between the stock and oil markets [10], concluding that the result of interaction between the international oil market and the international stock market has a one-way spillover effect on China's stock market. Dong Kun et al., studied whether it is possible to forecast the returns of China's stock market since 2003 through oil price changes [11]. They found that oil price changes do provide useful information to forecast the returns of China's stock market, which is significantly subject to the spillover effect of oil prices.

\section{MODELING}

\subsection{Copula Modeling}

According to Sklar's Theorem (Sklar, 1959) [12], let $x=\left(x_{1}, x_{2}\right)$, and $F, F_{1}$, and $F_{2}$ be distribution functions of $x, x_{1}, \mathrm{x}_{2}$ and respectively. Then $F$ may be expressed as 
$F(x)=C\left(F_{1}\left(x_{1}\right), F_{2}\left(x_{2}\right)\right)$, where $C$ is a cumulative distribution function (CDF) on $[0,1]^{2}$, called bivariate copula function.

If $F$ is absolutely continuous and $F_{1}, F_{2}$ are strictly increasing functions, then $F$ 's probability density function (PDF), i.e., $f$ may be expressed as:

$$
f(x)=c\left(F_{1}\left(x_{1}\right), F_{2}\left(x_{2}\right)\right) f_{1}\left(x_{1}\right) f_{2}\left(x_{2}\right)=c\left(u_{1}, u_{2}\right) f_{1}\left(x_{1}\right) f_{2}\left(x_{2}\right)
$$

Where,

$c$ is the PDF of $\mathrm{C}$;

$f_{1}, f_{2}$ is the PDF of $F_{1}, F_{2}$;

$u_{i}=F_{i}\left(x_{i}\right), i=1,2$.

There are many bivariate copula functions available, including Oval Copula, Archimedean Copulas and Mixed Copula. For the purpose of this paper, the bivariate copulas set includes Gaussian copula, Student-t copula, Clayton Copula, Gumbel copula, Frank copula, Joe copula, BB1 copula, BB6 copula, BB7 copula, and BB8 copula. Rotate Clayton copula, Gumbel copula, Joe copula, BB1 copula, BB6 copula, BB7 copula and BB8 copula by 90,180 and 270 degrees, and we can get the corresponding copula (A rotated copula (180 degrees) is called a survival copula. For example, a Joe copula rotated by 180 degrees is called Joe survival copula.). The Akaike Information Criterion (AIC) may be used to select the optimal copula from the above copula set through the following formula:

$A I C=-2 \sum_{i=1}^{n} \ln \left(c\left(u_{i, 1}, u_{i, 2} \mid \theta\right)\right)+2 k$

Where $k$ is the number of parameters in the copula function; $\theta$ is the parameter estimation of the copula function; and $\mathrm{n}$ is the sample size. After the identification of the optimal copula, we can use Kendall' $\tau$ to describe the non-linear dependence of two random variants $u_{1}, u_{2}$ through the following formula:

$\tau=4 \int_{[0,1]^{2}} C\left(u_{1}, u_{2}\right) d C\left(u_{1}, u_{2}\right)-1$.

In the meantime, the tail behaviors of $u_{1}, u_{2}$ may be described with the dependence coefficients of upper tail (UT) and Lower Tail (LT). UT dependence coefficient may be expressed as:

$\lambda_{U}=\lim _{u \rightarrow 1^{-}} \frac{1-2 u+C(u, u)}{1-u}$

and LT dependence coefficient as:

$\lambda_{L}=\lim _{u \rightarrow 0^{+}} \frac{C(u, u)}{u}$

\subsection{Copula-Based Calculation of VaR and ES}

In practice, two variants used extensively for risk measurement are Value at Risk (VaR) and Expected Shortfall (ES). They are calculated through the following formulas:

$$
\begin{aligned}
& V a R_{\alpha}=\inf \{l \in \mathbb{R}: P(-R>l) \leq 1-\alpha\} \\
& =\inf \left\{l \in \mathbb{R}: F_{-R}(l) \geq \alpha\right\}
\end{aligned}
$$

$E S=\frac{1}{1-\alpha} \int_{\alpha}^{1} \operatorname{VaR}_{u}(-R) d u$

Where,

$\alpha \in(0,1)$ is a given confidence level;

$R$ is log-return; and $F$ is the distribution function of loss $(-R)$.

As $F$ is usually unknown, it is impossible to calculate $\mathrm{VaR}$ and ES through the above formula (3), we can use Monte Carlo simulation based on the copula function to calculate $\mathrm{VaR}$ and ES through the following steps.

Given that $C\left(u_{1}, u_{2}, \theta\right)$ is a bivariate copula; that $\theta$ is its parameter vector; and that $v_{1}, v_{2}$ are two random samples subject to uniform distribution, then $u_{1}, u_{2}$ may be obtained through the following formula:

$u_{1}=v_{1}, u_{2}=h^{-1}\left(v_{2} \mid u_{1}, \theta\right)$

Where,

$h\left(v_{2} \mid u_{1}, \theta\right)=\frac{\partial C\left(v_{2}, u_{1}\right)}{\partial u_{1}}$, and $h^{-1}\left(v_{2} \mid u_{1}, \theta\right)$ is its inverse function.

Having obtained $u_{1}, u_{2}$, we can use

$z_{1}=G^{-1}\left(u_{1}\right), z_{2}=G^{-1}\left(u_{2}\right)$ to obtain $z_{1}, z_{2}$, (In this paper, $\mathrm{G}$ is a distribution function of shewed-t.) and then estimate VaR and ES with formula (3):

$$
\begin{aligned}
& V_{a R_{\mathrm{a}}{ }^{\prime}}=100 \times\left(\text { the } \mathrm{a}^{\text {th }} \text { empirical quantile of }-\mathrm{R}\right)(5) \\
& E S_{a}{ }^{\prime}=\text { the mean value of all }-\mathrm{Rs} \text { larger than } V R^{\prime}{ }_{a}
\end{aligned}
$$

\section{EMPIRICAL ANALYSIS OF RESULTS OBTAINED}

\subsection{Change-Point Tests from Data and Structure Per- spectives}

In this paper, the stock indexes of five developed markets, including DJIA, CAC40, DAX, FTSE100 and Nikkei 225 and three emerging markets, including Shanghai Stock Exchange Composite Index, India's BSE30 and Brazil's Bovespa in the time span of January 5, 2000 to March 15, 2013 were used. All stock index data were obtained from the RESSET database. All oil prices were as of WTI spot dates, as obtained from Energy Information Administration (EIA). WTI spot date oil prices are expressed in US\$/barrel, while in the RESSET database, stock index prices are indicated with RMB/point. To ensure consistency, the US\$/barrel was translated into $\mathrm{RMB} /$ barrel using the middle exchange rate between RMB and USD. To ensure consistency of trading days, if a particular index is not traded on a specific date (e.g., a statutory holiday, etc.), the data of other indexes on that date are deleted. This way, we collected data of 2,697 trading days. Then we use their log differences to obtain the return series.

During the recent years, a lot of structural change-point test methods have been proposed, including the Duel Segmentation, the Neighboring Structure Segmentation, the Bayesian Approach, and the PELT Approach. However, most of these approaches are useful only for structural 
change-point tests of single time series, not for multiple time series. Matteson and James proposed a nonparametric approach for multiple change point analysis of multivariate data. Another feature of this approach is that it uses few hypotheses [13]. In fact, the only hypothesis used here is there exists an absolute moment. The nonparametric approach uses the permutation test method to verify the significance of the multivariate data. The zero hypothesis of the permutation test is that there's no new structural change point. Through calculation, Matteson and James [13] found that, when permutation times $\mathrm{R}=499$ and $\mathrm{p}<0.05$, the zero hypothesis is rejected, indicating there's a significant new structural change point. Using Matteson and James [13] nonparametric approach, we conducted structural change point tests for the above $9 \log$ return series ( 8 stock markets and 9 oil price $\log$ return series) and obtained 4 common structural change points, all of which passed our significance test. See Table 1 for the results.

Table 1. Results of structural change point test.

\begin{tabular}{|c|c|c|c|c|}
\hline $\begin{array}{c}\text { Structural Change } \\
\text { Points }\end{array}$ & $\begin{array}{c}\mathbf{2 0 0 3 .} \\
\mathbf{5 . 2 0}\end{array}$ & $\begin{array}{c}\mathbf{2 0 0 7 .} \\
\mathbf{1 0 . 1 8}\end{array}$ & $\begin{array}{c}\mathbf{2 0 0 8} \\
\mathbf{. 9 . 2}\end{array}$ & $\begin{array}{c}\mathbf{2 0 0 9 .} \\
\mathbf{6 . 2}\end{array}$ \\
\hline \hline $\mathrm{P}$ & 0.002 & 0.002 & 0.002 & 0.018 \\
\hline
\end{tabular}

Each of the above structural change points corresponds to a significant incident in the financial market. For example, change point 2003.5.20 appeared after the US invasion of Iraq (March 20,2003), change point 2007.10.18 around the eruption of the financial crisis triggered by the subprime debt crisis; change point 2008.9.2 around the bankruptcy of Lehman Brothers, and change point 2009.6.2 around the release of regulatory capital management plan in the United States.

\subsection{Copula Functions Selection and Dependence Analysis}

The 4 structural change points in Table $\mathbf{1}$ divide the sample interval into 5 subintervals. We used copulas to analyze the dependence of oil price and the stock markets involved in each subinterval. First of all, we used the ARMA $(1,1)-$ GARCH $(1,1)$ to fit the oil prices with $\log$ return series of each market, obtaining its marginal distribution function through probability conversion of the resulting standard residuals. Then we used the copula models described in section 2 to select the right copula types and calculate Kendall' $\tau$ and tail dependence coefficients.

Fig. (1) illustrates the changes of Kendall' $\tau$ coefficient, which describes the dependence of oil and stock markets in each of the subintervals identified.

From Fig. (1) we can see that, Kendall' $\tau$ coefficients of the 2008/09/04-2009/06/02 time frame are significantly higher than those in the 2007/10/19-2008/09/02 time frame, indicating that the financial crisis in 2008 resulted in closer connections between stock and oil markets. In other words, it has enhanced the ability of stock and oil markets to jointly cope with risks. In the meantime, it also means that, with increased interaction between both markets, one is inevitably to fluctuate drastically along with the other.
Fig. (2) and Fig. (3) illustrate upper/lower tail dependence coefficient changes listed.

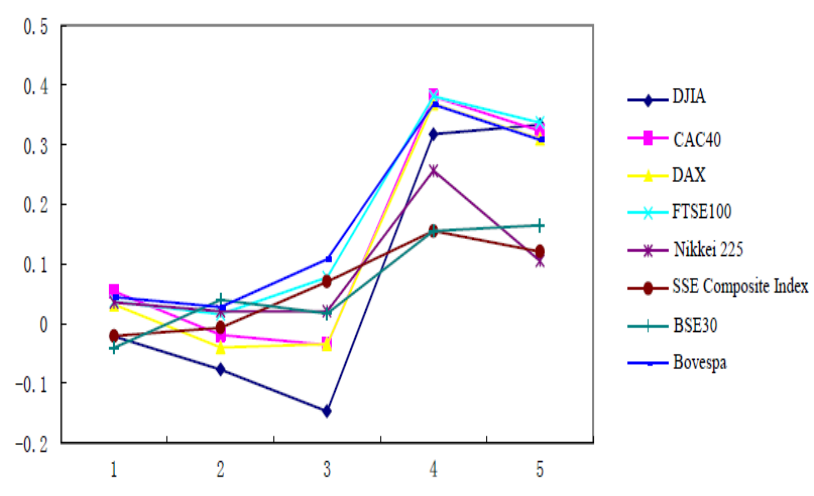

Fig. (1). Changes of Kendall' $\tau$ coefficient, which describes the dependence of oil and stock markets.

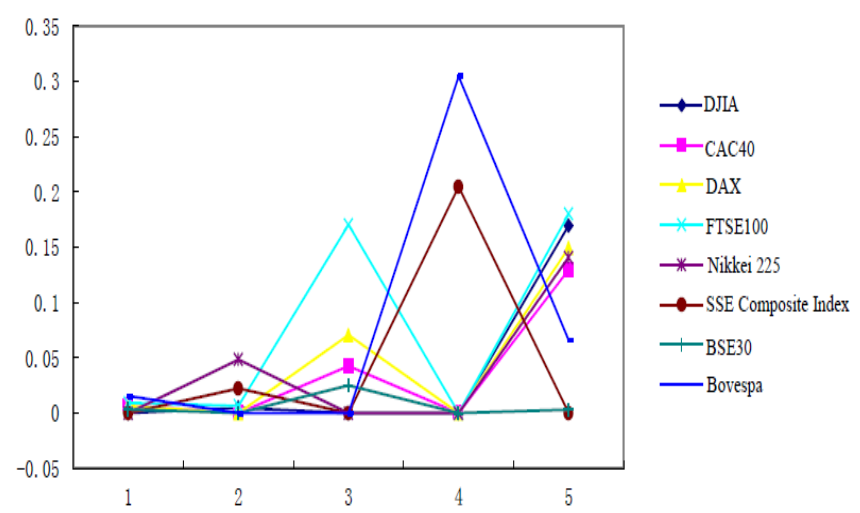

Fig. (2). Upper tail dependence coefficient changes of stock and oil markets.

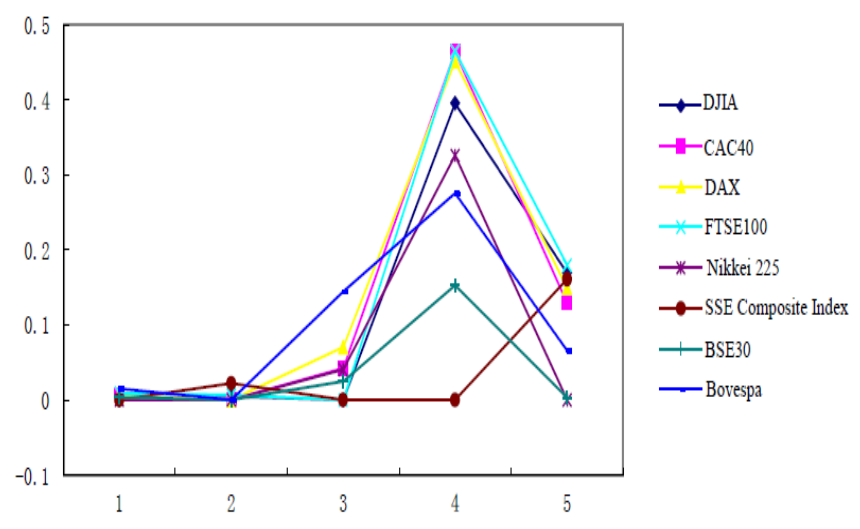

Fig. (3). Lower tail dependence coefficient changes of stock and oil markets.

From Fig. (2) and Fig. (3), we can see that, both upper and lower tail coefficients of the 2000/01/05-2003/05/20 and $2003 / 05 / 21-2007 / 10 / 18$ time frames are stable. In fact, most are equal to, or around zero. However, those of the 2007/10/19-2008/09/02 and 2008/09/04-2009/06/02 time frames are highly volatile. Particularly, lower tail coefficients of the 2008/09/04-2009/06/02 are significantly higher than the upper tail coefficients of same period, indicating stronger contamination between the oil and stock markets 
during the financial crisis. What should be noted is China's stock market seems to respond differently than other markets to oil market changes during the financial crisis. Its upper tail coefficient of the 2008/09/04-2009/06/02 is higher than its lower tail coefficient, indicating that, compared with other markets, China's stock market was less impacted by the financial crisis. It might have to do with a number of nonmarket factors, including lower levels of internationalization and market-oriented operation, and the intervention of the government.

Using the copula-based VaR and ES calculation approaches provided in Section 2, we obtained the 95\% quantile $\mathrm{VaR}$ and $\mathrm{ES}$ values of the log returns of the oil and stock market indexes.(Note: $95 \% \mathrm{VaR}$ and $95 \% \mathrm{ES}$ values of oil returns were obtained by fitting the oil price function with that of DJIA.)

Fig. (4) and Fig. (5) illustrate 95\%VaR and 95\%ES value changes.

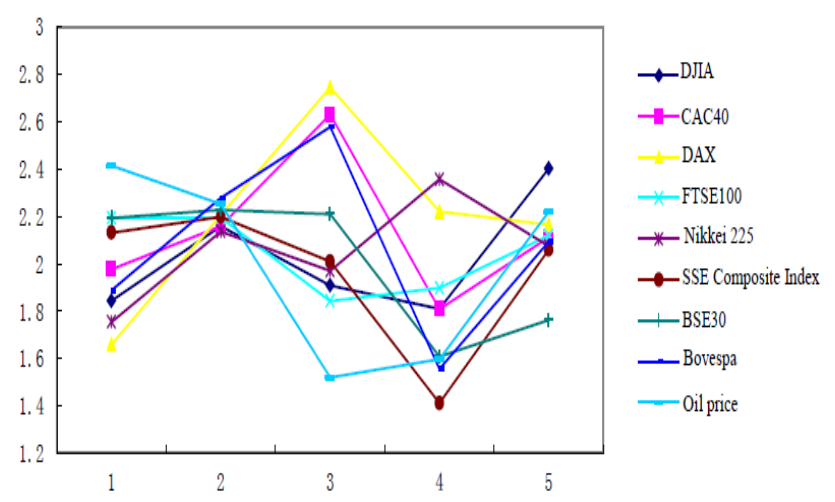

Fig. (4). $95 \% \mathrm{VaR}$ value changes of stock and oil markets.

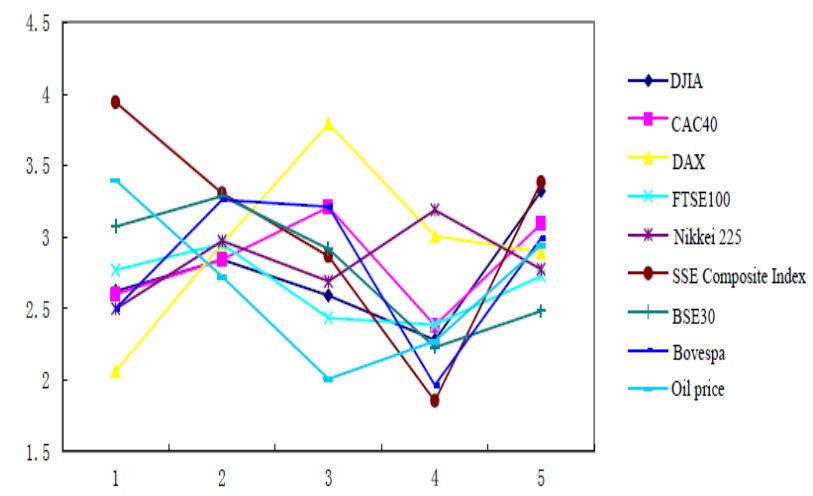

Fig. (5). 95\% ES value changes of stock and oil markets.

From Fig. (4 and 5) we can see that, in the sample interval, both oil and stock markets have been highly volatile. Compared with the stock market, the oil market has the highest $95 \%$ VaR value and the second highest $95 \%$ ES value in the first time span (2000/01/05-2003/05/20), indicating increased risk in the oil market due to the Iraqi war and other uncertainties. During the 2-3 time spans, risks in the oil market were declining, indicating that market panics were easing in the wake of the Iraqi war. Oil market risks rebounded in time spans 4-5, a proof of the significant impact of the financial crisis in 2008. With regard to the stock markets,
$95 \% \mathrm{VaR}$ and $95 \% \mathrm{ES}$ changes have been highly volatile, especially during the 4th time span (or the financial crisis), when developed markets, e.g., Japan, Germany and the United States had higher risks, while emerging markets, e.g., China and Brazil had medium/low risks. This indicates that, with lower level of internationalization, the emerging markets were less impacted by the shock in the international market in the midst of the financial crisis.

\section{CONCLUSION}

In the first step, using the nonparametric structural change point test approach, we tested the log returns of oil prices relative to DJIA, CAC40, DAX, FISE100, Nikkei 225, SSE Composite Index, BSE30 and Bovespa. The resulting 4 structural change points divided the sample interval (2000/01/05-2013/03/15) into 5 subintervals. Then we analyzed the dependence structure, $\mathrm{RaV}$ and ES of the oil and stock indexes of each subinterval. Key findings include:

Kendall' $\tau$ coefficients of stock and oil indexes during the financial crisis (2008-2009) are significantly higher than those before the crisis, indicating that the financial crisis in 2008 resulted in closer connections between stock and oil markets. In other words, it has enhanced the ability of stock and oil markets to jointly cope with risks. In the meantime, it also means that, with increased interaction between both markets, one is inevitably to fluctuate drastically along with the other. It requires investors to give sufficient consideration to the increasingly higher dependence, take better actions to prevent and avoid risk and maximize returns from oil futures or stock investments, or both.

Before the financial crisis, both upper and lower tail coefficients are very stable. In fact, most of them are equal to, or around zero. However, those during the financial crisis are highly volatile. Particularly, most lower tail coefficients of the period are significantly higher than the upper tail coefficients, indicating stronger contamination between the oil and stock markets during the financial crisis. What should be noted is China's stock market seems to respond differently than other markets to oil market changes during the financial crisis. Its upper tail coefficient of the 2008/09/04-2009/06/02 is higher than its lower tail coefficient, indicating that, compared with other markets, China's stock market was less impacted by the financial crisis. It might have to do with a number of non-market factors, including lower levels of internationalization and market-oriented operation, and the intervention of the government.

In the sample interval, both oil and stock markets have been highly volatile. Compared with the stock market, the oil market has the highest $95 \% \mathrm{VaR}$ value and the second highest $95 \%$ ES value in the 2000/01/05-2003/05/20 time frame, indicating increased risk in the oil market due to the Iraqi war and other uncertainties. During the 2003/05/212008/09/02 time frame, risks in the oil market were declining, indicating that market panics were easing in the wake of the Iraqi war. Oil market risks began to rebound in September 2008, a proof of the significant impact of the financial crisis in 2008. With regard to the stock markets, $95 \% \mathrm{VaR}$ and $95 \%$ ES changes have been highly volatile, especially during the 4th time span (or the financial crisis), when developed markets, e.g., Japan, Germany and the United States 
had higher risks, while emerging markets, e.g., China and Brazil had medium/low risks. It indicates that, with lower level of internationalization, the emerging markets were less impacted by the shock in the international market in the midst of the financial crisis.

\section{CONFLICT OF INTEREST}

The authors confirm that this article content has no conflict of interest.

\section{ACKNOWLEDGEMENTS}

The research work was supported by National Natural Science Foundation of China under Grant No. 71071022 and Tianjin Municipal Philosophy and Social Science Research Plan Project under Grant No. TJYY13-047.

\section{REFERENCES}

[1] N. Apergis, and S. M. Miller, "Do structural oil-market shocks affect stock prices," Energy Economics, vol. 31, no. 4, pp. 569-575, 2009.

[2] J. Park, and R. A. Ratti, "Oil price shocks and stock markets in the US and 13 European countries," Energy Economics, vol. 30, pp. 2578-2608, 2008

[3] M. Nandha, and R. Faff, "Does oil move equity prices? A global view ," Energy Economics, vol. 30, no. 3, pp. 986-997, 2008.
[4] L. Killian, and C. Park, "The impact of oil price shocks on the US stock market," International Economic Review, vol. 50, no. 4, pp. 1267-1287, 2009.

[5] S. Mohanty, M. Nandha, and G. Bota, "Oil shocks and stock returns: the case of the Central and Eastern European(CEE) oil and gas sectors," Emerging Markets Review, vol. 11, pp. 358-372, 2010.

[6] G. Driesprong, B. Jacobsen, and B. Matt, "Striling oil: Another puzzle," Journal of Financial Economics, vol. 89, pp. 307-327, 2008.

[7] H. Jin, and L. Jin, "Oil price and stock market spillover effect based on comparison and analysis of China and US market data," Financial Studies, vol. 332, no. 2, pp. 83-97, 2008.

[8] X. Liu, and C. Zhu, "Spillover effect of international oil price shocks on China's stock market," Journal of Guangdong University of Finance, no. 3, pp. 56-91, 2011.

[9] H. Jin, and L. Jin, "The impact of oil price on China's stock marketbased on empirical analysis of industrial data," Financial Studies, vol. 356, no. 2, pp. 173-187, 2010.

[10] D. Zhou, and J. Guo, "A bivariate VAR-GARCH $(1,1)$-BEKK model-based analysis on the spillover effect of financial and oil markets," Journal of China University of Petroleum (Edition of Natural Science), vol. 1, pp. 177-185, 2014.

[11] K. Dong, H. Xie, and S. Wang, "The myth about the connection between China's stock market and oil price changes," Journal of Management Science in China, vol. 11, pp. 45-53, 2012.

[12] A. Sklar, Fonctions Dé Repartition Á N Dimensions et Leurs Marges, Publications De l'Institut de Statistique de Paris, vol. 8, pp. 229-231, 1959.

[13] D.S. Matteson, and N.A. James, "A nonparameteic approach for multiple change point analysis of multivariate data," Journal of the American Statistical Association, vol. 109, no. 505, pp. 334-345, 2014.

Received: May 26, 2015

Revised: July 14, 2015

Accepted: August 10,2015

(C) Xin and Guofu; Licensee Bentham Open.

This is an open access article licensed under the terms of the (https://creativecommons.org/licenses/by/4.0/legalcode), which permits unrestricted, noncommercial use, distribution and reproduction in any medium, provided the work is properly cited. 